\title{
EDITORIAL
}

nature

cell biology

\section{Moving forward with cell migration}

Life has been infinitely easier post-PubMed. Recently joined by Scopus and Google Scholar, these essential tools allow access to specific information from the ever-expanding literature (see January editorial). They have limitations, however, when it comes to browsing, and reading around the field arguably provides the key to doing novel research. So how can you keep track of the papers relevant to your field, short of trawling through innumerable contents pages each month? Following the success of the Signaling Gateway, we are keen to develop new web resources to help researchers navigate through the literature jungle.

Cell migration is one field that continues to grow. To help you keep abreast, we have now launched the Cell Migration Gateway (www. cellmigration.org) - a collaboration between Nature Publishing Group (NPG) and the Cell Migration Consortium (CMC), which we hope will become the one-stop resource for what's what in this field. Focusing on topics from the basic mechanisms of motility to the different developmental and pathological processes that migration impinges on, the aim is to collate and discuss new advances in this broad field, providing a much required compass for the community.

The CMC was established in September 2001 with funding from a National Institute of General Medical Sciences (NIGMS) 'glue grant'. Its aim is to develop novel technologies, data and information that will help unravel the mechanisms of cell migration. Driven by Rick Horwitz and Tom Parsons at the University of Virginia, the consortium is based on a collaboration of around 40 investigators from 20 institutions working together on six initiatives: the cell migration proteome, structures, modelling, imaging, transgenics and expression screens. A primary aim of the CMC is to disseminate its fruits rapidly; to this end, results generated from these initiatives will go online as soon as is feasible in the CMC Activity Center.

Complementing these research initiatives is the Cell Migration Knowledgebase designed by Bill Pearson, also at Virginia. The aim is to collate and organize data generated by CMC, and to incorporate this with published literature to provide a key fact file for each molecule relevant to cell migration research; so far, more than 900 molecules are covered by the database. The Knowledgebase records will link to the CMC Activity Center and other databases such as UniProt, OMIM and the AfCS/Nature Molecule Pages.

The Cell Migration Gateway directs users to both the CMC Activity Center and the CM Knowledgebase. In addition, it also contains a third section generated by NPG editors - Cell Migration Update. Through editorial summaries of papers and a research library, we will provide access to essential discoveries in cell migration research as they are published each month. Keywording aids browsability and indeed a primary aim of this site is to aid reading across the increasingly diverse landscape of migration research. Because papers from key journals across the board will be covered, it should provide a broad overview of the field. We hope this will ensure that users of the site do not miss vital papers, and are kept informed about more divergent aspects of the field. We also aim to inform you of new additions to the CMC Activity Center periodically, so that opportunities waiting to be exploited are not missed.

\section{Databases in peril}

While we launch CMG as a long-term community tool, other consortiums and databases are facing a tough future. In Europe, database funding is often linked to research projects. Europe has nurtured a significant number of databases of global impact, such as Swiss-Prot; and the EBI in Cambridge continues to be a focal point for cutting edge bioinformatics. However, a database can only thrive when it is well maintained with regular data-feeds. It is lamentable that European governmental funding does not usually secure the long-term sustainability of databases and several worthwhile projects are facing an uncertain future as a result (see Nature, 23 June). Indeed, some databases have become commercial as a result; this can constrain access to the data, as was seen all too clearly when the Yeast Protein Database became part of the commercial entity BIOBASE.

While the NIH is the main supporter of the umbrella protein database UniProt (which contains the European databases SwissProt and TrEMBL) and provides funds for a number of key organismal databases such as FlyBase, all is not so well on the other side of the Atlantic. The Canadian government, for example, insists that its funding be matched by independent sources, which has led to the cancellation of funding for a number of large-scale projects (see Science, 24 June). One such project is the BIND database (see August 2004 editorial), which is set to shift activities to Singapore as a result (Science 308, 1529; 2005). Another project is the US-based Alliance for Cell Signaling (AfCS), which faces a sizable reduction for the second term of their NIGMS 'glue grant'. Aside from curtailing the data acquisition part of the project, this has necessitated the move to acquire alternative funding to continue expansion of the Molecule Pages database and the Signaling Gateway (see www.signaling-gateway.org/).

Evidently databases are proliferating and some redundancy is emerging. However, no more so than with bench research, and some overlap is to be encouraged to ensure projects improve in a competitive environment. The information explosion in the biological sciences necessitates that funding agencies invest as much in the initiation of these database projects as in their long-term future, which is usually a fraction of the initial cost. It is hoped that funding agencies can transcend national boundaries and traditional funding criteria to ensure that the best databases become permanent research tools only this will allow a full exploitation of the promise of systems biology. 